\title{
On Explaining Integer Vectors by Few Homogenous Segments
}

\author{
Robert Bredereck ${ }^{\star}$, Jiehua Chen ${ }^{\star \star}$, Sepp Hartung, Christian Komusiewicz, \\ Rolf Niedermeier, and Ondřej Suchý ${ }^{\star \star \star}$ \\ Institut für Softwaretechnik und Theoretische Informatik, TU Berlin \\ \{robert.bredereck, jiehua.chen, sepp.hartung, christian.komusiewicz, \\ rolf.niedermeier\}@tu-berlin.de \\ Faculty of Information Technology, Czech Technical University in Prague \\ ondrej.suchy@fit.cvut.cz
}

\begin{abstract}
We extend previous studies on NP-hard problems dealing with the decomposition of nonnegative integer vectors into sums of few homogeneous segments. These problems are motivated by radiation therapy and database applications. If the segments may have only positive integer entries, then the problem is called VECTOR ExPLANATION ${ }^{+}$. If arbitrary integer entries are allowed in the decomposition, then the problem is called Vector Explanation. Considering several natural parameterizations (including maximum vector entry, maximum difference between consecutive vector entries, maximum segment length), we obtain a refined picture of the computational (in-)tractability of these problems. In particular, we show that in relevant cases VECTOR ExplanATION ${ }^{+}$is algorithmically harder than VECTOR EXPLANATION.
\end{abstract}

\section{Introduction}

We study two variants of a "mathematically fundamental" [4], NP-hard combinatorial problem occurring in cancer radiation therapy planning [10] and database and data warehousing applications $[1,18]$ :

Vector Explanation (VECTOR Explanation ${ }^{+}$)

Input: A vector $A \in \mathbb{N}^{n}$ with $A[1]>0$ and $A[n]>0$ and an integer $k$.

Question: Can $A$ be explained by at most $k$ (positive) segments?

Herein, a segment is a $0 / a$-vector, $a \in \mathbb{Z} \backslash\{0\}$, with $n$ entries where all $a$-entries occur consecutively, and it is positive if $a$ is positive. An explanation is a set of segments that sum up to the input vector. For instance, in case of VECTOR Explanation (VE for short) the vector $(4,3,3,4)$ can be explained by the segments $(4,4,4,4)$ and $(0,-1,-1,0)$, and in case of VeCTOR Explanation ${ }^{+}$ ( $\mathrm{VE}^{+}$for short) it can be explained by $(3,3,3,3),(1,0,0,0)$, and $(0,0,0,1)$.

^ Supported by the DFG, research project PAWS, NI 369/10.

$\star \star$ Supported by the Studienstiftung des Deutschen Volkes.

$\star \star \star$ The main work was done while O. Suchý was at TU Berlin, supported by the DFG, research project AREG, NI 369/9.

To appear in Proceedings of the 13th Algorithms and Data Structures Symposium - WADS'13, London, Ontario, Canada, August 2013. (c) Springer. 
Table 1. An overview of previous and new results.

\begin{tabular}{|c|c|c|}
\hline Parameters & VECTOR EXPLANATION & VECTOR EXPLANATION $^{+}$ \\
\hline $\max$. value $\gamma$ & \multirow{3}{*}{ fpt (Thm. 2(2)) } & $\begin{array}{c}2^{O(\sqrt{\gamma})} \cdot \gamma n[6] \\
\text { no poly. kernel (Thm. 3) }\end{array}$ \\
\hline $\begin{array}{c}\text { max. difference } \delta \text { of } \\
\text { two consecutive entries }\end{array}$ & & $O\left(n^{\delta+1} \cdot e^{\pi \sqrt{2 \delta / 3}}\right)($ Thm. 2(3)) \\
\hline$(\#$ of peaks $p, \delta)$ & & fpt (Thm. 2(1)) \\
\hline number $k$ of segments & \multicolumn{2}{|c|}{$\begin{array}{c}k^{O(k)}+n^{O(1)}(\mathrm{Thm} .4) \\
(2 k-1) \text {-entry kernel (Thm. 4) }\end{array}$} \\
\hline$k^{\prime}=2 k-n$ & $\begin{array}{c}k^{\prime O\left(k^{\prime}\right)}+n^{O(1)}(\text { Thm. } 5(3)) \\
\left.3 k^{\prime} \text {-entry kernel (Thm. } 5(3)\right)\end{array}$ & $\begin{array}{c}k^{O\left(k^{\prime}\right)}+n^{O(1)}(\text { Thm. 5(1)) } \\
\mathrm{W}[1] \text {-hard }(\text { Thm. 5(2)) }\end{array}$ \\
\hline$n-k$ & \multicolumn{2}{|c|}{ NP-hard for $(n-k)=1($ Thm. $6(2))$} \\
\hline max. segment length $l$ & \multicolumn{2}{|c|}{$\begin{array}{c}l \geq 3: \text { NP-hard }(\text { Thm. } 6(1)) \\
l \leq 2: O\left(n^{2}\right)(\text { Thm. } 6(2))\end{array}$} \\
\hline $\begin{array}{c}\text { max. number } o \text { of } \\
\text { overlapping segments }\end{array}$ & \multicolumn{2}{|c|}{$\begin{array}{c}o=1: \text { trivial } \\
o=2(\text { and } l=3 \text { and } n-k=1): \text { NP-hard (Thm. 6(1)) }\end{array}$} \\
\hline
\end{tabular}

VE occurs in the database context and $\mathrm{VE}^{+}$occurs in the radiation therapy context. Motivated by previous work providing polynomial-time solvable special cases $[1,4]$, polynomial-time approximation $[5,19]$ and fixed-parameter tractability results $[6,8]$ (approximation and fixed-parameter algorithms both exploit problem-specific structural parameters), we head on a systematic parameterized and multivariate complexity analysis $[13,21]$ of both problems; see Table 1 for a survey of parameterized complexity results.

Previous work. Agarwal et al. [1] studied a polynomial-time solvable variant ("tree-ordered") of VE relevant in data warehousing. Karloff et al. [18] initiated a study of (special cases of) the two-dimensional ("matrix") case of VE and provided NP-hardness results as well as polynomial-time constant-factor approximations. Parameterized complexity aspects of VE and its two-dimensional variant seem unstudied so far.

The literature on $\mathrm{VE}^{+}$is richer. For a general account on the motivation from radiation therapy refer to the survey by Ehrgott et al. [10]. Concerning computational complexity, $\mathrm{VE}^{+}$is known to be strongly NP-hard [3] and APX-hard [4]. A significant amount of work has been done to achieve approximation algorithms for minimizing the number of segments which improve on the straightforward factor of two [4] (also see Biedl et al. [5]). Improving a previous fixed-parameter tractability result for the parameter "maximum value $\gamma$ of a vector entry" by Cambazard et al. [8], Biedl et al. [6] developed a fixed-parameter algorithm solving $\mathrm{VE}^{+}$in polynomial time when $\gamma=O\left((\log n)^{2}\right)$ with $n$ being the number of entries in the input vector. Moreover, the parameter "maximum difference between two consecutive vector entries" has been exploited for developing polynomial-time 
approximation algorithms [5, 19]. Finally, we remark that most of the previous studies also looked at the two-dimensional ("matrix") case, whereas we focus on the one-dimensional ("vector") case.

Our contributions. We observe that the combinatorial structure of the considered problems is extremely rich, opening the way to a more thorough study of the computational complexity landscape under the perspective of problem parameterization. We take a closer look at these parameterization aspects that help in better understanding and exploiting problem-specific properties. To start with, note that previous work $[6,8]$, motivated by the application in radiation therapy, studied the parameterization by the maximum vector entry $\gamma$. They showed fixed-parameter tractability for $\mathrm{VE}^{+}$parameterized by $\gamma$, which we complement by showing the non-existence (assuming a standard complexity-theoretic assumption) of a corresponding polynomial-size problem kernel. Using an integer linear program (ILP) formulation, we also show fixed-parameter tractability for VE parameterized by $\gamma$. Moreover, for the perhaps most obvious parameter, the number $k$ of explaining segments, we show fixed-parameter tractability for both problems. In addition, we study the following parameters:

Definition 1. For an input vector $A \in \mathbb{N}^{n}$ where, for notational convenience, additionally $A[0]=A[n+1]=0$ define:

- the maximum difference $\delta$ between two consecutive vector entries ( $\delta=$ $\left.\max _{1 \leq i \leq n+1}|A[i]-A[i-1]|\right)$;

- the number $p$ of peaks (a position $1 \leq i \leq n$ is a peak if $A[i-1]<A[i]>$ $A[i+1])$;

- the maximum segment length $l$ (number of $a$-entries);

- the maximum number $o$ of segments having a non-zero entry at a particular vector entry;

- "distance from triviality"-parameter $n-k$;

- "distance from triviality"-parameter $k^{\prime}:=2 k-n$.

Concerning the parameters $n-k$ and $k^{\prime}$, a brief discussion is appropriate. As to $n-k$, note that the problems have trivial solutions if $k=n$ : just take $n$ segments with one non-zero entry each. In this sense, $n-k$ is a parameterization by "distance from triviality" [16, 21]. We show that, somewhat surprisingly, both problems are already NP-hard for $k=n-1$. As to $k^{\prime}$, note that by a simple preprocessing which will be explained later on, we can achieve that for every resulting instance which is not already classified as no-instance, we have that $n \leq 2 k-1 .^{1}$ Moreover, if $k=\lfloor n / 2\rfloor+1$, then the instances are polynomial-time solvable, motivating the "distance from triviality-parameter" $k$ '. Interestingly, while we show that $\mathrm{VE}^{+}$is $\mathrm{W}[1]$-hard for parameter $k^{\prime}$, we show that $\mathrm{VE}$ is fixed-parameter tractable for $k^{\prime}$. Finally, we show NP-hardness for $l=3$ and $o=2$.

${ }^{1}$ The definition of $k^{\prime}$ refers to the number $n$ of entries in the instance after the preprocessing. 
Table 1 summarizes our and previous results. Our work is organized as follows. In Section 2, we present a number of useful combinatorial properties of vector explanation problems which may be of independent interest and which are used throughout our work. In Section 3, we study the "smoothness of input vector" parameters $\gamma, \delta$, and $p$. In Section 4, we present results for further parameters as discussed above, and we conclude with some challenges for future research. Due to the lack of space most proofs and details are deferred to a full version.

Parameterized complexity preliminaries. A parameterized problem is fixedparameter tractable (fpt) if all instances $(I, k)$ consisting of the "classical" problem instance $I$ and the parameter $k$ can be solved in $f(k) \cdot|I|^{O(1)}$ time for any function $f$ solely depending on $k$. A kernelization algorithm is a polynomialtime algorithm that transforms each instance $(I, k)$ for a problem $L$ into an instance $\left(I^{\prime}, k^{\prime}\right)$ for $L$ such that $(I, k) \in L \Leftrightarrow\left(I^{\prime}, k^{\prime}\right) \in L$ (equivalence) and $k^{\prime},\left|I^{\prime}\right| \leq g(k)$ for some function $g$. The instance $\left(I^{\prime}, k^{\prime}\right)$ is called a (problem) kernel of size $g(k)$. A kernelization algorithm is often described by a set of data reduction rules whose exhaustive application leads to a problem kernel. An instance is called reduced with respect to a data reduction rule if a further application would have no effect on the instance.

A problem that is shown to be W[1]-hard by means of a parameterized reduction from a W[1]-hard problem is not fpt, unless FPT $=\mathrm{W}[1]$. A parameterized reduction maps an instance $(I, k)$ in $f(k) \cdot|I|^{O(1)}$ time to an equivalent instance $\left(I^{\prime}, k^{\prime}\right)$ with $k^{\prime} \leq g(k)$ for some functions $f$ and $g$. See [20] for a more detailed introduction to parameterized algorithmics. We assume the unit-cost RAM model where arithmetic operations on numbers count as a single computation step.

\section{Combinatorial Properties}

Formally, for an input vector $A \in \mathbb{N}^{n}$ a segment $I$ is a pair written as $[l, r]$ with $l, r \in\{1,2, \ldots, n+1\}$ and $l<r$. We say $I$ starts at position $l$ and ends at positions $r$. A segment $[l, r]$ covers position $i$ whenever $l \leq i<r$. A set of segments $\mathcal{I}$, together with a weight function $w: \mathcal{I} \rightarrow \mathbb{Z} \backslash\{0\}$, forms an explanation for $A \in \mathbb{N}^{n}$ if

$$
\forall 1 \leq i \leq n: A[i]=\sum_{I \in \mathcal{I} \text { covers } i} w(I),
$$

where $A[i]$ denotes the $i$ th entry in $A$. We also say $(\mathcal{I}, w)$ explains $A$. In case of $\mathrm{VE}^{+}$, we only allow positive weights, that is, $w: \mathcal{I} \rightarrow \mathbb{N} \backslash\{0\}$. We refer to $|\mathcal{I}|$ as solution size. Segments with positive weight are called positive segments, those with negative weight are called negative segments.

Definition 2. A position $1 \leq i \leq n+1$ with respect to a vector $A \in \mathbb{N}^{n}$, is called an uptick if $A[i-1]<A[i]$ and called downtick if $A[i-1]>A[i]$. The size of an uptick (resp. downtick) $i$ equals $|A[i]-A[i-1]|$.

By the following known data reduction rule [4], we may assume that each position is either an uptick or a downtick. 
Rule 1 If vector $A$ has two consecutive equal entries, then remove one of them.

Rule 1 can be applied exhaustively in $O(n)$ time. Afterwards, each position in $A$ is either an uptick or a downtick. By the following lemma, we can assume that in solutions for $\mathrm{VE}^{+}$the segments start in upticks and end in downticks.

Lemma 1 ([4, Lemma 1]). Let $(A, k)$ be an instance of $\mathrm{VE}^{+}$. There is a minimum-size explanation for vector $A$ in which each segment starts at an uptick and ends at a downtick.

For VE, we can generalize Lemma 1 to hold for negative and positive segments. Actually, one can even "reorder" all consecutive up- and downticks. This implies that for VE actually only the sizes of the upticks and downticks matter, not their order. To formalize this, we introduce the notion of single-peakedness.

Definition 3. A vector is single-peaked if it contains only one peak. A singlepeaked instance is an instance with a single-peaked vector.

The following theorem summarizes combinatorial properties of $\mathrm{VE}$ and $\mathrm{VE}^{+}$ which are used throughout the paper and which may be of independent interest.

Theorem 1. Let $(A, k)$ be an instance of VE. Then, the following holds.

1. There is a minimum-size explanation for $(A, k)$ in which each positive segment starts at an uptick and ends at a downtick, and each negative segment starts at a downtick and ends at an uptick.

2. For any position $1 \leq i \leq n$ setting $A[i] \leftarrow A[i-1]+A[i+1]-A[i]$ results in an equivalent instance.

3. If $(A, k)$ is single-peaked, then $(A, k)$ is an equivalent $\mathrm{VE}^{+}$instance.

4. The instance $(A, k)$ can be reduced in $O\left(n+k^{2}\right)$ time to an equivalent singlepeaked instance $\left(A^{\prime}, k\right)$ such that the maximum difference between consecutive entries is the same in $A$ and $A^{\prime}$.

5. There is an equivalent instance $\left(A^{\prime}, k\right)$ with $A^{\prime} \in\{0, \ldots, 2 \delta-1\}^{n}$ where $\delta$ is the maximum difference between consecutive entries of $A$.

Further, the following holds for $\mathrm{VE}^{+}$and for single-peaked VE instances.

6. There is a minimum-size explanation such that

(a) there is only one segment, starting at an uptick, that covers the last entry and

(b) there is only one segment, ending at a downtick, that covers the first entry.

7. If an instance $(A, k)$ is a yes-instance, then $A$ contains at most $k$ upticks and at most $k$ downticks.

\section{Parameterization by Input Smoothness}

In this section, we examine how the computational complexity of $\mathrm{VE}$ and $\mathrm{VE}^{+}$ is influenced by parameters that measure how "smooth" the input vector $A \in \mathbb{N}^{n}$ is. We assume that $A$ is reduced with respect to Rule 1 and thus all consecutive 
positions in $A$ have different values. We consider the following three measurements: the maximum difference $\delta$ between two consecutive values in $A$, the number $p$ of peaks, that is, the number of positions $1 \leq i \leq n$ in $A$ such that $A[i-1]<$ $A[i]>A[i+1]$, and the maximum value $\gamma$ occurring in $A$. Our main results are fixed-parameter algorithms for the combined parameter $(p, \delta)$ in the case of $\mathrm{VE}^{+}$and for the parameter $\delta$ in the case of VE. For the parameter maximum value $\gamma$, we show that $\mathrm{VE}^{+}$does not admit a polynomial-size problem kernel unless $\mathrm{NP} \subseteq$ coNP/poly.

Theorem 2. 1. $\mathrm{VE}^{+}$parameterized by the combined parameter number $p$ of peaks and maximum difference $\delta$ is fixed-parameter tractable.

2. VE parameterized by the maximum difference $\delta$ or the maximum value $\gamma$ is fixed-parameter tractable.

3. $\mathrm{VE}^{+}$is solvable in $O\left(n^{\delta+1} \cdot e^{\pi \sqrt{2 \delta / 3}}\right)$ time.

Proof. We only prove the correctness of Theorem 2(1). This also implies Theorem 2(2): By Theorem 1(3) and Theorem 1(4), we can transform input instances of $\mathrm{VE}$ into single-peaked ones of $\mathrm{VE}^{+}$without increasing the maximum difference $\delta$. Furthermore, $\delta \leq \gamma$. Together with the above transformation this implies fixed-parameter tractability for $\delta$ and for $\gamma$. The proof of Theorem 2(3) is based on a dynamic programming algorithm, omitted from this extended abstract.

To show Theorem 2(1), we provide an integer linear program (ILP) formulation for $\mathrm{VE}^{+}$where the number of variables is a function of $p$ and $\delta$. This ILP determines whether there is a size- $k$ solution with the properties given by Lemma 1 , that is, a solution in which each segment starts at an uptick and ends at a downtick. In such a solution, the multiset of weights of segments that start at an uptick sum up to the uptick size. This analogously holds for segments ending at a downtick. Motivated by this fact, we introduce the following notion: For a positive integer $x$, we say that a multiset $X=\left\{x_{1}, x_{2}, \ldots, x_{r}\right\}$ of positive integers partitions $x$ if $x=\sum_{i=1}^{r} x_{i}$. Similarly, we say that $X$ partitions an uptick (downtick) $i$ of size $x$ if $X$ partitions $x$. Let $\mathcal{P}(x)$ denote the set of all multisets that partition $x$.

In the ILP, we describe a solution by "fixing" for each $i \in\{1, \ldots, n\}$ a multiset $X_{i}$ of positive integers which partitions the uptick (downtick) at $i$. The crucial observation for our ILP is that if a set of consecutive upticks contains more than one uptick of size $x$, it is sufficient to fix how many of these upticks were partitioned in which way. In other words, one does not need to know the partition for each position; instead one can distribute freely the partitions of $x$ onto the upticks of size $x$. This also holds for consecutive downticks. Since each peak is preceded by consecutive upticks and succeeded by consecutive downticks, and since we introduce variables in the ILP formulation to "model" how many upticks (downticks) exist between two consecutive peaks, the number of variables in the formulation is bounded by a function of $p$ and $\delta$. We now give the details of the formulation. Herein, we assume that the peaks are ordered from left to right; we refer to the $i$-th peak in this order as peak $i$.

For an integer $x \in\{1, \ldots, \delta\}$, let $\operatorname{occ}(x, i)$ denote the number of upticks of size $x$ that directly precede peak $i$, that is, the number of upticks succeeding 
peak $i-1$ and preceding peak $i$. Similarly, let occ $(-x, i)$ denote the number of downticks of size $x$ that directly succeed $i$. For two positive integers $y$ and $x$ with $y \leq x$ and a multiset $P \in \mathcal{P}(x)$ let mult $(y, P)$ denote how often $y$ appears in $P$. We use mult $(y, P)$ to "model" how many segments of weight $y$ start (end) at some uptick (downtick) that is partitioned by $P$.

To formulate the ILP, we introduce for each peak $i$, each $x \in\{1, \ldots, \delta\}$, and each $P \in \mathcal{P}(x)$ two nonnegative variables $\operatorname{var}_{x, P, i}$ and $\operatorname{var}_{-x, P, i}$. The variables correspond to the number of upticks directly preceding peak $i$ and downticks directly succeeding peak $i$ of size $x$ that are partitioned by $P$ in a possible explanation of $A$. To enforce that a particular assignment to these variables corresponds to a valid explanation, we introduce the following constraints.

First, for each peak $i$ and each $1 \leq x \leq \delta$ we ensure that the number of directly proceeding size- $x$ upticks (succeeding size- $x$ downticks) that are partitioned by some $P \in \mathcal{P}(x)$ is equal to the number of directly proceeding size- $x$ upticks (succeeding size- $x$ downticks):

$$
\forall i \in\{1, \ldots, p\}, \forall x \in\{-\delta, \ldots, \delta\} \backslash\{0\}: \sum_{P \in \mathcal{P}(x)} \operatorname{var}_{x, P, i}=\operatorname{occ}(x, i) .
$$

Second, we ensure that for each peak $i$ and each value $y \in\{1, \ldots, \delta\}$ the number of segments of weight $y$ that end directly after peak $i$ is at most the number of segments of weight $y$ that start at positions (not necessarily directly) preceding peak $i$ minus the number of segments of weight $y$ that end at positions succeeding some peak $j<i$. Informally, this means that we only "use" the available number of segments of weight $y$. To enforce this property, for each peak $1 \leq i \leq p$ and each possible segment weight $1 \leq y \leq \delta$ we add:

$$
\sum_{j=1}^{i} \sum_{x=y}^{\delta} \sum_{P \in \mathcal{P}(x)}(\underbrace{\operatorname{mult}(y, P) \cdot \operatorname{var}_{x, P, j}}_{\# \text { of opened weight- } y \text { segments }}-\underbrace{\operatorname{mult}(y, P) \cdot \operatorname{var}_{-x, P, j}}_{\# \text { of closed weight- } y \text { segments }}) \geq 0
$$

Finally, we ensure that the total number of segments is at most $k$ :

$$
\sum_{i=1}^{p} \sum_{x=1}^{\delta} \sum_{P \in \mathcal{P}(x)} \sum_{y=1}^{x} \operatorname{mult}(y, P) \cdot \operatorname{var}_{x, P, i} \leq k .
$$

Correctness: The equivalence of the ILP instance and $(A, k)$ can be seen as follows. Assume that there is a size-at-most- $k$ explanation $S$ for $(A, k)$. Recall that by definition of $\mathcal{P}(x)$, for any uptick $i$ of size $x$ there is a partition in $\mathcal{P}(x)$ that corresponds to the weights of the segments starting in $i$. For each peak $i$, for any value $1 \leq x \leq \delta$ and each $P \in \mathcal{P}(x)$, count how many upticks of size $x$ that directly precede peak $i$ are explained by segments in $S$ (segments that start in this uptick) whose weights correspond to $\mathcal{P}(x)$ and set $\operatorname{var}_{x, P, i}$ to this value. Symmetrically, do the same for the downticks succeeding the peak $i$ and set $\operatorname{var}_{-x, P, i}$ accordingly. It is straightforward to verify that eqs. (1) to (3) hold.

Reversely, assume that there is an assignment to the variables such that eqs. (1) to (3) are fulfilled. We form a set of segments $S$ as follows: For any 
peak $i$ and any value $1 \leq x \leq \delta$ with $\operatorname{occ}(x, i)>0$ let $\mathcal{P}_{i, x}$ be the multiset of partitions of $\mathcal{P}(x)$ that contains each $P \in \mathcal{P}(x)$ exactly $\operatorname{var}_{x, P, i}$ times. By eq. (1), $\left|\mathcal{P}_{i, x}\right|=\operatorname{occ}(x, i)$. For an arbitrary ordering of $\mathcal{P}_{i, x}$ and the upticks of size $x$ directly preceding peak $i$, add to $S$ for the $j$ th element $\mathcal{P}_{j}$ of $\mathcal{P}_{i, x}$ exactly $\left|\mathcal{P}_{j}\right|$ segments with weight corresponding to $\mathcal{P}_{j}$ and let them start at the $j$ th uptick with size $x$ that directly precedes peak $i$. By eq. (3) we added at most $k$ segments. It remains to specify the end of the segments. Symmetrically to the upticks, for each downtick directly succeeding peak $i$ of size $x$ let $\mathcal{P}_{i, x}$ be the multiset of elements from $\mathcal{P}(x)$ containing each $P \in \mathcal{P}(x)$ exactly $\operatorname{var}_{-x, P, i}$ times. For the $j$ th element $\mathcal{P}_{j}$ of $\mathcal{P}_{i, x}$ and the $j$ th downtick directly succeeding peak $i$ (again both with respect to any ordering) and for each $\alpha \in \mathcal{P}_{j}$ pick any weight- $\alpha$ segment from $S$ (so far without end) and let it end directly one position behind the $j$ th downtick. Observe that the existence of this segment is ensured by eq. (2). Finally, it remains to argue that the of end of each segment in $S$ is determined. This follows from the fact that eqs. (1) and (2) together imply for each $1 \leq y \leq \delta$ that

$$
\sum_{i=1}^{p} \sum_{x=y}^{\delta} \sum_{P \in \mathcal{P}(x)}\left(\operatorname{mult}(y, P) \cdot \operatorname{var}_{x, P, i}-\operatorname{mult}(y, P) \cdot \operatorname{var}_{-x, P, i}\right)=0,
$$

and thus the total number of opened weight- $y$ segments is equal to the number of closed weight- $y$ segments.

Running time: The ILP can be solved within the following time bound. The number of variables in the constructed ILP instance is

$$
p \cdot \sum_{x \in\{-\delta, \ldots, \delta\} \backslash\{0\}}|\mathcal{P}(|x|)|=2 p \sum_{x=1}^{\delta}|\mathcal{P}(x)| \leq 2 \delta p \cdot|\mathcal{P}(\delta)| \leq 2 \delta p \cdot e^{\pi \sqrt{\frac{2}{3} \delta}}=: f(\delta, p),
$$

where the last inequality is due to de Azevedo Pribitkin [2]. Thus, due to a deep result in combinatorial optimization the feasibility of the ILP can decided in $O\left(f(\delta, p)^{2.5 f(\delta, p)+o(f(\delta, p))} \cdot|L|\right)$ time, where $|L|$ is the size of the instance [14, 17]. Moreover, as we have $O(\delta p)$ inequalities, we also have $|L|=O\left(\delta^{2} p^{2} \cdot e^{\pi \sqrt{\frac{2}{3} \delta}}\right)$.

For the parameter maximum value $\gamma, \mathrm{VE}^{+}$is known to be fixed-parameter tractable [6]. We complement this result by showing a lower bound on the problem kernel size, and thus demonstrate limitations on the power of preprocessing.

Theorem 3. Unless $N P \subseteq$ coNP/poly, there is no polynomial-size problem kernel for $\mathrm{VE}^{+}$parameterized by the maximum value $\gamma$.

Proof. We provide a so-called AND-cross-composition [7, 9] from the 3-PARTITION problem. Given a multiset $S=\left\{a_{1}, \ldots, a_{3 m}\right\}$ of positive integers and an integer bound $B$ with $m \cdot B=\sum_{i=1}^{3 m} a_{i}$ and $B / 4<a_{i}<B / 2$ for every $i \in\{1, \ldots, 3 m\}, 3$-PARTITION asks whether the set $S$ can be partitioned into $m$ subsets $P_{1}, \ldots, P_{m}$ with $\left|P_{j}\right|=3$ and $\sum_{a_{i} \in P_{j}} a_{i}=B$ for every $j \in\{1, \ldots, m\} .3$ PARTITION is NP-hard even if $B$ (and thus all $a_{i}$ 's) is bounded by a polynomial in 
$m$ [15]. We show that this variant of 3-PARTITION AND-cross-composes to $\mathrm{VE}^{+}$ parameterized by the maximum value $\gamma$. Then, results of Bodlaender et al. [7] and Drucker [9] imply that $\mathrm{VE}^{+}$does not have a polynomial-size problem kernel with respect to parameter $\gamma$, unless $\mathrm{NP} \subseteq$ coNP/poly.

First, let $(S, B)$ be a single instance of 3-PARTition. We show that it reduces to an instance $\left(A^{\prime}, 3 m\right)$ of $\mathrm{VE}^{+}$. This reduction is very similar to a previous NP-hardness reduction for $\mathrm{VE}^{+}[4]$. We define $A^{\prime}$ as length- $(4 m-1)$ vector:

$$
\left(a_{1}, a_{1}+a_{2}, \ldots, \sum_{i=1}^{j} a_{i}, \ldots, \sum_{i=1}^{3 m} a_{i}=m B,(m-1) B,(m-2) B, \ldots, B\right) .
$$

On the one hand, if a partition $P_{1}, \ldots, P_{m}$ of $S$ forms a solution, then the set of segments $\left\{[i, 3 m+j] \mid a_{i} \in P_{j}\right\}$ each with weight $w([i, 3 m+j])=a_{i}$ is an explanation for the vector $A^{\prime}$. On the other hand, let $(\mathcal{I}, w)$ be an explanation for $\left(A^{\prime}, 3 m\right)$. By Lemma 1 we may assume that every segment starts at an uptick and ends at a downtick. Therefore, $\mathcal{I}$ contains $3 m$ segments and the segment starting at position $i$ has weight $a_{i}$. Since $B / 4<a_{i}<B / 2$ for each integer $a_{i} \in S$, exactly three segments end at a downtick whose size is exactly $B$. Thus, grouping the segments according to the position they end at, we get the desired partition of $S$, solving the instance of 3-PARTITION.

Now let $\left(S_{1}, B_{1}\right), \ldots,\left(S_{t}, B_{t}\right)$ be instances of 3-PARTition such that $S_{r}=$ $\left\{a_{1}^{r}, \ldots, a_{3 m_{r}}^{r}\right\}$ and $B_{r} \leq m_{r}{ }^{c}$ for every $r \in\{1, \ldots, t\}$ and some constant $c$. We build an instance $(A, k)$ of $\mathrm{VE}^{+}$by first using the above reduction for each $\left(S_{r}, B_{r}\right)$ separately to produce a vector $A_{r}^{\prime}$, and then concatenating the vectors $A_{r}^{\prime}$ one after another, leaving a single position of value 0 in between. The total length of the vector $A$ is $4\left(\sum_{r=1}^{t} m_{r}\right)-1$ and we set $k:=3 \sum_{r=1}^{t} m_{r}$.

Due to the argumentation for the single instance case, on the one hand, if each of the instances is a yes-instance, then there is an explanation using $3 m_{r}$ segments per instance $\left(S_{r}, B_{r}\right)$, that is $3 \sum_{r=1}^{t} m_{r}$ segments in total. On the other hand, we need at least $3 m_{r}$ segments to explain $A_{r}^{\prime}$ and there is an explanation with $3 m_{r}$ segments if and only if $\left(S_{r}, B_{r}\right)$ is a yes-instance. Since all segments are positive and the subvectors $A_{r}^{\prime}$ 's are separated by a position with value zero, no segment can span over two subvectors. In other words, no segment can be used to explain more than one of the $A_{r}^{\prime}$ 's. Therefore, an explanation for $A$ with $3 \sum_{r=1}^{t} m_{r}$ segments implies that $\left(S_{r}, B_{r}\right)$ is a yes-instance for every $r \in\{1, \ldots, t\}$.

Finally, observe that the maximum value $\gamma$ in the vector $A$ is equal to $\max _{r=1}^{t} m_{r} B_{r} \leq \max _{r=1}^{t} m_{r}{ }^{c+1}$. Since in each input 3-PARTITION instance the maximum value $m_{r} B_{r}$ is polynomially bounded in the instance size $\left|S_{r}\right|$, this value is thus polynomially bounded in $\max _{r=1}^{t}\left|S_{r}\right|$. Hence, 3-PARTITION ANDcross-composes to $\mathrm{VE}^{+}$parameterized by the maximum value $\gamma$, and there is no polynomial-size problem kernel for this problem unless $\mathrm{NP} \subseteq$ coNP/poly.

\section{Further Parameterizations}

We now provide fixed-parameter tractability and (parameterized) hardness results for further natural parameters. Specifically, we consider the number $k$ of 
segments in the solution, so-called "above-guarantee" and "below-guarantee" parameterizations (which are smaller than $k$ ), the maximum segment length $l$, and the maximum number of segments covering a position.

For the parameter $k$ we obtain fixed-parameter tractability by using Rule 1 , Theorem 1(6), and Theorem 1(7) to develop search tree algorithms for $\mathrm{VE}^{+}$and VE. The depth and the branching degree of the search tree are bounded by the solution size $k$. The second part of Theorem 4 follows directly from exhaustively applying Rule 1.

Theorem 4. $\mathrm{VE}^{+}$and $\mathrm{VE}$ can be solved in $O(k ! \cdot k+n)$ time. Any instance of $\mathrm{VE}^{+}$or $\mathrm{VE}$ can be reduced in $O(n)$ time to an equivalent one with at most $(2 k-1)$ entries.

The second part of Theorem 4 implies that for a reduced instance every explanation needs at least $\lfloor n / 2\rfloor+1$ segments. Furthermore, instances with $k=\lfloor n / 2\rfloor+1$ are solvable in polynomial time (below, we will state a generalization of this fact). Hence, it is interesting to study parameters that measure how far we have to exceed this lower bound for the solution size; notably, such above-guarantee parameters can be significantly smaller than $k$. For this reason, we study a parameter that measures $k-\lfloor n / 2\rfloor-1$. For ease of presentation, we define this parameter as $k^{\prime}:=2 k-n$. The concepts of "clean" and "messy" positions, which are defined as follows, are crucial for the design of our algorithms.

Definition 4. Let $(A, k)$ be an instance of $\mathrm{VE}$ or $\mathrm{VE}^{+}$and let $\mathcal{I}$ be an explaining segment set for vector $A$. A segment $I=[i, j] \in \mathcal{I}$ is clean if all other segments start and end at positions different from $i$ and $j$. A position $i$ is clean with respect to $\mathcal{I}$ if it is the start or endpoint of a clean segment in $\mathcal{I}$. A position or segment that is not clean is called messy.

We show that clean positions can always be covered by clean segments of "minimum length": Iterate from left to right over all clean positions and for each position $i$ (still clean) find the first clean position $j>i$ with $-(A[i]-A[i-1])=$ $A[j]-A[j+1]$ and add a segment of weight $A[i]-A[i-1]$ from $i$ to $j+1$.

For every yes-instance of $\mathrm{VE}$, the number of messy positions is at most $3 k^{\prime}$ and the number of messy segments used by an explanation is at most $2 k^{\prime}$ with $k^{\prime}=2 k-n$. Furthermore, if there are an uptick and a downtick of same size in a single-peaked instance, then we may assume that the corresponding segment is contained in the solution.

As the following theorem shows, using the properties concerning clean and messy positions, we can replace the exponent $k$ in the running time of Theorem 4 by the smaller $k^{\prime}$. This also implies that $\mathrm{VE}^{+}$is polynomial-time solvable for constant $k^{\prime}$. Unless $\mathrm{W}[1]=\mathrm{FPT}$, this result cannot be improved to fixed-parameter tractability since we can give a parameterized reduction from the W[1]-hard Subset Sum problem [11] to $\mathrm{VE}^{+}$. In contrast, $\mathrm{VE}^{+}$for single-peaked instances as well as VE in general are fixed-parameter tractable with respect to $k^{\prime}$ and can be efficiently reduced to equivalent instances with at most $3 k^{\prime}$ positions.

Theorem 5. 1. $\mathrm{VE}^{+}$can be solved in $O\left((2 k)^{3 k^{\prime}} \cdot\left(k^{2}+\left(2 k^{\prime}\right) ! \cdot k^{\prime}\right)+n\right)$ time. 
2. $\mathrm{VE}^{+}$is $W[1]$-hard with respect to $k^{\prime}$.

3. Any single-peaked instance of $\mathrm{VE}^{+}$and any instance of $\mathrm{VE}$ can be reduced in $O\left(k^{2}+n\right)$ time to an equivalent one with most $3 k^{\prime}$ entries. Moreover, $\mathrm{VE}^{+}$ and VE are solvable in $O\left(\left(2 k^{\prime}\right) ! \cdot k^{\prime}+k^{2}+n\right)$ time.

The previous parameter $k^{\prime}$ measures how far the solution exceeds the lower bound $\lfloor n / 2\rfloor+1$. Another bound on the solution size is $n$ : If $k=n$, then any instance of $\mathrm{VE}^{+}$or $\mathrm{VE}$ is a trivial yes-instance. Hence, it is interesting to consider the parameter $n-k$. Furthermore, it is natural to consider explanations with restricted segment length $l$ or the maximum number $o$ of segments overlapping at some position. The following theorem shows that $\mathrm{VE}^{+}$and $\mathrm{VE}$ are already NP-hard even if $k=n-1, l \geq 3$, and $o=2$. To this end, we reduce from the NP-hard PARTITION problem [15]. In terms of parameterized complexity this implies that, unless $\mathrm{P}=\mathrm{NP}, \mathrm{VE}^{+}$is not fixed-parameter tractable with respect to the "maximum segment length $l$ ", the "maximum number $o$ of segments overlapping at some position", and the "below guarantee parameter" $n-k$.

We also show that, in contrast to the NP-hardness for $l \geq 3, \mathrm{VE}^{+}$and $\mathrm{VE}$ are polynomial-time solvable for $l \leq 2$.

Theorem 6. 1. $\mathrm{VE}^{+}$and $\mathrm{VE}$ are NP-hard even if $k=n-1$ and every yesinstance has an explanation of at most $k$ segments where each position is covered by at most two segments and each segment has length at most three.

2. Both $\mathrm{VE}^{+}$and $\mathrm{VE}$ can be solved in $O\left(n^{2}\right)$ time for maximum segment length $l=2$.

\section{Conclusion}

It would be interesting to significantly improve on several of the running time upper bounds of our (theoretical) tractability results (cf. Table 1 for an overview). Moreover, we also left open a number of concrete problems. We conclude with three of them:

- Is $\mathrm{VE}^{+}$fixed-parameter tractable with respect to the maximum difference $\delta$ ?

- Does VE parameterized by $\delta$ or parameterized by $\gamma$ admit a polynomial-size problem kernel?

- Is $\mathrm{VE}$ or $\mathrm{VE}^{+}$fixed-parameter tractable with respect to the parameter "number of different values in the input vector $A$ "? This parameter would be a natural version of "parameterization by the number of numbers" [12].

Acknowledgement: We are very grateful for the very detailed and constructive feedback provided by the WADS reviewers.

\section{Bibliography}

[1] D. Agarwal, D. Barman, D. Gunopulos, N. Young, F. Korn, and D. Srivastava. Efficient and effective explanation of change in hierarchical summaries. In Proc. 13th KDD, pages 6-15. ACM, 2007. 
[2] W. de Azevedo Pribitkin. Simple upper bounds for partition functions. The Ramanujan Journal, 18:113-119, 2009.

[3] D. Baatar, H. W. Hamacher, M. Ehrgott, and G. J. Woeginger. Decomposition of integer matrices and multileaf collimator sequencing. Discrete Appl. Math., 152 (1-3):6-34, 2005.

[4] N. Bansal, D. Z. Chen, D. Coppersmith, X. S. Hu, S. Luan, E. Misiolek, B. Schieber, and C. Wang. Shape rectangularization problems in intensity-modulated radiation therapy. Algorithmica, 60(2):421-450, 2011.

[5] T. C. Biedl, S. Durocher, H. H. Hoos, S. Luan, J. Saia, and M. Young. A note on improving the performance of approximation algorithms for radiation therapy. Inf. Process. Lett., 111(7):326-333, 2011.

[6] T. C. Biedl, S. Durocher, C. Engelbeen, S. Fiorini, and M. Young. Faster optimal algorithms for segment minimization with small maximal value. Discrete Appl. Math., 161(3):317-329, 2013.

[7] H. L. Bodlaender, B. M. P. Jansen, and S. Kratsch. Cross-composition: A new technique for kernelization lower bounds. In Proc. 28th STACS, volume 9 of LIPIcs, pages 165-176. Schloss Dagstuhl-Leibniz-Zentrum fuer Informatik, 2011.

[8] H. Cambazard, E. O'Mahony, and B. O'Sullivan. A shortest path-based approach to the multileaf collimator sequencing problem. Discrete Appl. Math., 160(1-2): 81-99, 2012.

[9] A. Drucker. New limits to classical and quantum instance compression. In Proc. 53rd IEEE FOCS, pages 609-618. IEEE Computer Society, 2012.

[10] M. Ehrgott, C. Güler, H. Hamacher, and L. Shao. Mathematical optimization in intensity modulated radiation therapy. Ann. Oper. Res., 175:309-365, 2010.

[11] M. R. Fellows and N. Koblitz. Fixed-parameter complexity and cryptography. In Proc. 10th AAECC, volume 673 of LNCS, pages 121-131. Springer, 1993.

[12] M. R. Fellows, S. Gaspers, and F. A. Rosamond. Parameterizing by the number of numbers. Theory Comput. Syst., 50(4):675-693, 2012.

[13] M. R. Fellows, B. M. P. Jansen, and F. A. Rosamond. Towards fully multivariate algorithmics: Parameter ecology and the deconstruction of computational complexity. European J. Combin., 34(3):541-566, 2013.

[14] A. Frank and É. Tardos. An application of simultaneous diophantine approximation in combinatorial optimization. Combinatorica, 7(1):49-65, 1987.

[15] M. R. Garey and D. S. Johnson. Computers and Intractability: A Guide to the Theory of NP-Completeness. Freeman, 1979.

[16] J. Guo, F. Hüffner, and R. Niedermeier. A structural view on parameterizing problems: Distance from triviality. In Proc. 1st IWPEC, volume 3162 of LNCS, pages 162-173. Springer, 2004.

[17] R. Kannan. Minkowski's convex body theorem and integer programming. Math. Oper. Res., 12:415-440, 1987.

[18] H. Karloff, F. Korn, K. Makarychev, and Y. Rabani. On parsimonious explanations for 2-d tree- and linearly-ordered data. In Proc. 28th STACS, volume 9 of LIPIcs, pages 332-343. Schloss Dagstuhl-Leibniz-Zentrum fuer Informatik, 2011.

[19] S. Luan, J. Saia, and M. Young. Approximation algorithms for minimizing segments in radiation therapy. Inf. Process. Lett., 101(6):239-244, 2007.

[20] R. Niedermeier. Invitation to Fixed-Parameter Algorithms. Oxford University Press, 2006.

[21] R. Niedermeier. Reflections on multivariate algorithmics and problem parameterization. In Proc. 27th STACS, volume 5 of LIPIcs, pages 17-32. Schloss Dagstuhl-Leibniz-Zentrum fuer Informatik, 2010. 


\section{A Appendix}

\section{A.1 Proof of Theorem 1}

Theorem 1(1) Let $(A, k)$ be an instance of VE. Then, there is a minimum-size explanation in which each positive segment starts at an uptick and ends at a downtick and each negative segment starts at a downtick and ends at an uptick.

Proof. Suppose that there is no minimum-size solution obeying the stated conditions. We say that a segment $I \in \mathcal{I}$ has a wrong start if $I$ is positive and starts at a downtick or if it is negative and starts at an uptick. Otherwise, we say the start is correct. We define wrong ends similarly. A segment is wrong if it has either wrong start or wrong end.

Among all minimum-size explanations of $A$, let $(\mathcal{I}, w)$ be one without wrong end, or, if there is no such explanation, then let $(\mathcal{I}, w)$ be such that its first wrong end is on the leftmost position. Moreover, among these explanations, let $(\mathcal{I}, w)$ be one without wrong start or one where the first wrong start is on the rightmost position.

Assume now that there is a segment with a wrong start and let $I$ be the segment with the wrong start on the earliest position. We distinguish two cases. First, let $I=\left[i, i^{\prime}\right]$ be positive and $i$ be a downtick. Since $i$ is a downtick, there is a segment $J$ such that either $J$ is positive and ends at $i$, or $J$ is negative and starts at $i$.

Case 1: $J$ is positive and ends at $i$. By the choice of $I$, the interval $J$ has a correct start, say at position $j$. If $w(J) \geq w(I)$, then we replace $I$ by segment $I^{\prime}=\left[j, i^{\prime}\right]$, set $w\left(I^{\prime}\right)=w(I)$, and decrease the weight of the segment $J$ by $w(I)$ or remove $J$ if $w(J)=w(I)$. It is easy to see that the new set of segments still explains $A$ and, as $I^{\prime}$ has correct start, the total weight of segments with wrong start at $i$ is decreased. As the signs of the weights are kept and $I^{\prime}$ ends where $I$ ended, no new wrong end is introduced. If $w(J)<w(I)$, then replace $J$ by $J^{\prime}=\left[j, i^{\prime}\right]$ and decrease the weight of $I$ by $w(J)$. Again, $A$ is still explained, no new (position with a) wrong end is introduced and the total weight of segments with wrong start at $i$ is decreased.

Case 2: $J$ is negative and starts at $i$. Let $J=\left[i, j^{\prime}\right]$. If $j^{\prime}<i^{\prime}$, then distinguish three subcases. If $-w(J)>w(I)$, then replace $I$ by $I^{\prime}=\left[j^{\prime}, i^{\prime}\right]$ with weight $w\left(I^{\prime}\right)=w(I)$ and increase the weight of $J$ by $w(I)$. For $-w(J)=w(I)$ use the same replacement for $I$ and remove $J$. Finally, if $-w(J)<w(I)$, then replace $J$ by a positive segment $J^{\prime}=\left[j^{\prime}, i^{\prime}\right]$ with weight $-w(J)$, and decrease the weight of $I$ by $-w(J)$. If $i^{\prime}=j^{\prime}$, then use the same replacements as above, just omit the segments of zero length. If $j^{\prime}>i^{\prime}$ and $-w(J)>w(I)$ then replace $I$ by a negative segment $I^{\prime}$ with weight $-w(I)$ starting at $i^{\prime}$ and ending on $j^{\prime}$ and increase the weight of $J$ by $w(I)$. If $-w(J)=w(I)$, then use the same replacement for $I$ and remove $J$. Finally, if $-w(J)<w(I)$, then replace $J$ by $J^{\prime}$ with weight $w\left(J^{\prime}\right)=w(J)$, which starts in $i^{\prime}$ and ends in $j^{\prime}$ and decrease the weight of $I$ by $-w(J)$. It is easy to check that in all cases the modified segments still explain $A$, and the total weight of segments with wrong start at $i$ is decreased. Moreover, none of the new segments starts before $i$ and whenever a positive segment is 
introduced, it ends where previously a positive segment ended, and a negative segment ends where also previously a negative segment ended.

Since $(\mathcal{I},-w)$ is an explanation for $-A$ with the same properties as $(\mathcal{I}, w)$, the case of $I$ being negative and starting at an uptick, can be solved by applying the above transformations to $(\mathcal{I},-w)$.

Therefore, we can apply the above modifications as long as there is a segment with wrong start at $i$, each time decreasing the total absolute value of weight of segments with wrong start at $i$. Hence, after a finite number of modifications, there is no segment with a wrong start at $i$. Since we never introduce a segment with a wrong start before $i$ or a new wrong end, this contradicts the choice of $(\mathcal{I}, w)$. Hence we can assume that in $(\mathcal{I}, w)$ there is no segment with wrong start.

Now let $A^{\prime}$ be the vector obtained by reversing the vector $A$ and $\mathcal{I}^{\prime}$ the set of segments obtained by reversing each segment in $\mathcal{I}$. As $\mathcal{I}$ has no wrong start, $\mathcal{I}^{\prime}$ has no wrong end. Applying the above argumentation to $\left(\mathcal{I}^{\prime}, w\right)$ we can show that $\mathcal{I}^{\prime}$ also has no wrong start. The property of having no wrong end is preserved by the modifications. Therefore, also $\mathcal{I}$ has no wrong start, no wrong end, all segments are correct and the claim follows.

Theorem 1(2) For any position $i \in\{2, \ldots, n-1\}$ setting $A[i] \leftarrow A[i-1]+A[i+$ $1]-A[i]$ results in an equivalent instance.

Proof. Let $A^{\prime}[j]=A[j]$ for every $j \neq i$ and $A^{\prime}[i]=A[i-1]+A[i+1]-A[i]$. We prove that $\left(A^{\prime}, k\right)$ is a yes-instance if and only if $(A, k)$ is a yes-instance.

Note first that if we define $A^{\prime \prime}$ as $A^{\prime \prime}[j]=A^{\prime}[j]$ for every $j \neq i$ and $A^{\prime \prime}[i]=$ $A^{\prime}[i-1]+A^{\prime}[i+1]-A^{\prime}[i]$, then we have $A^{\prime \prime}[i]=A[i-1]+A[i+1]-(A[i-1]+$ $A[i+1]-A[i])=A[i]$ and $A^{\prime \prime}=A$. Hence, the second direction of the equivalence is symmetric to the first one and it is thus sufficient to prove that if $(A, k)$ is a yes instance, then so is $\left(A^{\prime}, k\right)$.

Let $(\mathcal{I}, w)$ be a size- $k$ solution for $A$. We construct $\left(\mathcal{I}^{\prime}, w^{\prime}\right)$ by replacing some segments in $\mathcal{I}$. The general idea is that if a segment started or ended at position $i$, then it should now start or end at $i+1$ and vice versa. The only exception are the segments which start at $i$ and end at $i+1$. Namely $\mathcal{I}^{\prime}$ is defined as follows:

$$
\begin{aligned}
& \mathcal{I}^{\prime}=\mathcal{I}_{1}^{\prime} \cup \mathcal{I}_{2}^{\prime} \cup \mathcal{I}_{3}^{\prime} \cup \mathcal{I}_{4}^{\prime} \cup \mathcal{I}_{5}^{\prime} \cup \mathcal{I}_{6}^{\prime}, \text { where } \\
& \mathcal{I}_{1}^{\prime}=\{[a, b] \in \mathcal{I} \mid a \notin\{i, i+1\}, b \notin\{i, i+1\}\}, \\
& \mathcal{I}_{2}^{\prime}=\{[a, i+1] \mid[a, i] \in \mathcal{I}\}, \\
& \mathcal{I}_{3}^{\prime}=\{[a, i] \mid[a, i+1] \in \mathcal{I}, a \leq i-1\}, \\
& \mathcal{I}_{4}^{\prime}=\{[i+1, b] \mid[i, b] \in \mathcal{I}, b \geq i+2\}, \\
& \mathcal{I}_{5}^{\prime}=\{[i, b] \mid[i+1, b] \in \mathcal{I}\}, \\
& \mathcal{I}_{6}^{\prime}=\{[i, i+1]\} \cap \mathcal{I} .
\end{aligned}
$$

We let $w^{\prime}([i, i+1])=-w([i, i+1])$ if $[i, i+1] \in \mathcal{I}$, and for the other segments of $\mathcal{I}^{\prime}$ we set the weight $w^{\prime}$ to be equal to the corresponding segment in $\mathcal{I}$.

Obviously, $\left|\mathcal{I}^{\prime}\right|=|\mathcal{I}|$ and, hence, it remains to show that $\left(\mathcal{I}^{\prime}, w^{\prime}\right)$ explains $A^{\prime}$. As a segment of $\mathcal{I}^{\prime}$ covers a position $j \neq i$ of $A^{\prime}$ if and only if the corresponding 
segment of $\mathcal{I}$ of the same weight covers the position in $A$, it is clear that $\left(\mathcal{I}^{\prime}, w^{\prime}\right)$ explains every position $A^{\prime}[j]=A[j]$ with $j \neq i$.

Let $\mathcal{I}_{0}^{\prime}=\left\{[a, b] \mid[a, b] \in \mathcal{I}_{1}^{\prime}, a \leq i-1, b \geq i+2\right\}$. Since $(\mathcal{I}, w)$ explains $A$, and because of the way we defined $\mathcal{I}^{\prime}$ and $w^{\prime}$, we have $A[i-1]=s_{0}+s_{2}+s_{3}$, $A[i]=s_{0}+s_{3}+s_{4}-s_{6}$, and $A[i+1]=s_{0}+s_{4}+s_{5}$, where $s_{x}=\sum_{I \in \mathcal{I}_{x}^{\prime}} w^{\prime}(I)$. The sum of the weights of segments covering $A^{\prime}[i]$ is $s_{0}+s_{2}+s_{5}+s_{6}$. In addition, we have $A^{\prime}[i]=A[i-1]+A[i+1]-A[i]=s_{0}+s_{2}+s_{3}+s_{0}+s_{4}+s_{5}-\left(s_{0}+s_{3}+s_{4}-s_{6}\right)=$ $s_{0}+s_{2}+s_{5}+s_{6}$. Therefore, $\left(\mathcal{I}^{\prime}, w^{\prime}\right)$ also explains $A^{\prime}[i]$.

Theorem 1(3) Let $A$ be a single-peaked vector. Then, $(A, k)$ is a yes-instance for $\mathrm{VE}$ if and only if $(A, k)$ is also a yes-instance for $\mathrm{VE}^{+}$.

Proof. Let $(A, k)$ be a single-peaked yes-instance of VE and consider the solution provided by Theorem 1(1) (possibly using negative weights). As $A$ is singlepeaked, no segment can start in a downtick and end in an uptick. Therefore all segments are positive and $(A, k)$ is a also a yes-instance of $\mathrm{VE}^{+}$. Clearly, in the other direction, it is obvious that a solution for $\mathrm{VE}^{+}$is also a valid solution for VE.

Using the modification specified by Theorem 1(2), one can significantly simplify the structure of VE instances.

Theorem 1(4) An instance $(A, k)$ of VE can be reduced in $O\left(n+k^{2}\right)$ time to an equivalent single-peaked instance $\left(A^{\prime}, k\right)$ such that the maximum difference between consecutive entries is the same in $A$ and $A^{\prime}$.

Proof. Assume that the instance is reduced with respect to Rule 1. The reduction works as follows. If $A$ is single-peaked, then nothing has to be done. Otherwise, find the leftmost position $i$ with $A[i-1]>A[i]<A[i+1]$ (call such a position a dent) and perform the modification of Theorem 1(2). The running time of exhaustively applying these modifications is $O\left(k^{2}\right)$ : First, finding such a position can be done in $O(k)$ time. After the application of the modification, check whether position $A^{\prime}[i-1]$ is now a dent. If this is the case, then apply the modification to $A^{\prime}[i-1]$. Otherwise, $A^{\prime}[i]$ is now the leftmost peak. That is, the position of the leftmost peak has increased by one. Hence, in $O(k)$ time one can apply the modification such that the position of the leftmost peak has increased. After $O(k)$ repetitions of this procedure, the instance has to be single-peaked. The overall running time follows.

Concerning the maximum difference $\delta$, observe that after one application of the modification in Theorem $1(2)$ we have $A^{\prime}[i]-A^{\prime}[i-1]=A[i-1]+A[i+1]-A[i]-$ $A[i-1]=A[i+1]-A[i]$, while $A^{\prime}[i+1]-A^{\prime}[i]=A[i+1]-A[i-1]-A[i+1]+A[i]=$ $A[i]-A[i-1]$. Hence, the maximum of the differences between $A[i]$ and its neighbor positions remains the same.

Theorem 1(5) For every instance $(A, k)$ of $\mathrm{VE}$, there is an equivalent instance $\left(A^{\prime}, k\right)$ with $A^{\prime} \in\{0, \ldots, 2 \delta-1\}^{n}$ where $\delta$ is the maximum difference between consecutive values of $A$. 
Proof. By Theorem 1(2), one obtains equivalent instances by arbitrarily reordering the upticks and downticks. We can compose $A^{\prime}$ in such a way that if $A[i]<\delta$, then the position $i+1$ is an uptick and otherwise it is a downtick. As the size of each uptick and each downtick is at most $\delta$, it follows that in this way $0 \leq A^{\prime}[i] \leq 2 \delta-1$ for every $i$.

The following observation proves that in case of $\mathrm{VE}^{+}$or in case of a singlepeaked instance of VE the solution can be assumed to be well-structured.

Theorem 1(6) For instances $(A, k)$ of $\mathrm{VE}^{+}$and for single-peaked instances of $\mathrm{VE}$ there is a minimum-size explanation such that

(a) there is only one segment starting at an uptick that covers the last entry and

(b) there is only one segment ending at a downtick that covers the first entry.

Proof. The proofs of (a) and (b) work analogously. Hence, we only prove (a).

Note that we can assume w.l.o.g. that all segments start at an uptick, end at a downtick, and are positive: For $\mathrm{VE}^{+}$, this follows directly from Lemma 1. In VE instances with only one peak there is no $i<j$ such that $A[i]$ is a downtick and $A[j]$ is an uptick. Hence, by Theorem 1(1) there is a solution without negative segments. Let $\mathcal{I}$ be a set of a most $k$ segments explaining $A$ and let $Z:=\left\{\left[s_{1}, n+1\right],\left[s_{2}, n+1\right], \ldots,\left[s_{z}, n+1\right]\right\}$ with the upticks $s_{1} \leq s_{2} \leq \ldots \leq s_{z}$ denote the set of segments from $\mathcal{I}$ covering the last entry $A[n]$. We define $x:=\left[s_{z}, n+1\right]$ with $\omega(x)=\sum_{i=1}^{z} \omega\left(\left[s_{i}, n+1\right]\right)=A[n]$ and $Y:=\left\{\left[s_{i}, s_{z}\right] \mid i<z\right\}$ with $\omega\left(\left[s_{i}, s_{z}\right]\right)=\omega\left(\left[s_{i}, n+1\right]\right), 1 \leq i \leq z-1$. Now, $\mathcal{I}^{*}:=(\mathcal{I} \cup\{x\} \cup Y) \backslash Z$ explains $A$ and $\left|\mathcal{I}^{*}\right|=k+1+(z-1)-z=k$.

Theorem 1(7) It follows directly from Lemma 1 and Theorem 1(1).

\section{A.2 The Remaining Proof of Theorem 2}

It is open whether $\mathrm{VE}^{+}$is fixed-parameter tractable with respect to $\delta$. Note that Theorem 2(1) and Theorem 2(2) cannot be transferred, since there may be more than one peak in a given instance. However, the following theorem implies that $\mathrm{VE}^{+}$can be solved in polynomial time if the maximum difference $\delta$ is a constant.

Theorem 2(3) $\mathrm{VE}^{+}$is solvable in $O\left(n^{\delta+1} \cdot e^{\pi \sqrt{\frac{2}{3} \delta}}\right)$ time.

Proof. We describe a dynamic programming algorithm that finds a minimum-size solution that has the properties given in Lemma 1. Every explanation for a size- $n$ vector $A$ can be interpreted as an extension of an explanation for the same vector without the last entry, where some segments that originally only covered position $n-1$ are stretched to also cover position $n$ and some new segment of length one may start at position $n$.

Our algorithm uses the above relation between explanations for the vector $A[1, \ldots, n]$ and explanations for the vector $A[1, \ldots, n-1]$. Due to Lemma 1 , it only considers explanations where each segment starts at an uptick and ends 
at a downtick. Since all upticks and downticks have size at most $\delta$, the algorithm furthermore only considers solutions in which all segments have weight at most $\delta$.

The dynamic programming table has entries of the type $T\left(i, d_{1}, \ldots, d_{j}, \ldots, d_{\delta}\right)$ where $0 \leq i \leq n, 0 \leq d_{j} \leq n$, and $1 \leq j \leq \delta$. An entry $T\left(i, d_{1}, \ldots, d_{j}, \ldots, d_{\delta}\right)$ contains the minimum number of segments explaining vector $A[1, \ldots, i]$ such that $d_{j}$ segments of weight $j$ cover position $i$. If no such explanation exists, then the entry is set to $\infty$. By definition of the table entries, there is a solution for $\mathrm{VE}^{+}$ if and only if

$$
\min _{\left(d_{1}, \ldots, d_{\delta}\right) \in\{0, \ldots, n\}^{\delta}} T\left(n, d_{1}, \ldots, d_{\delta}\right) \leq k .
$$

Now, we show how to fill the table. As initialization, set $T\left(0, d_{1}, \ldots, d_{\delta}\right) \leftarrow \infty$ for all $\left(d_{1}, \ldots, d_{\delta}\right) \in\{0, \ldots, n\}^{\delta}$ with $d_{j}>0$ for any $j \in\{1, \ldots, \delta\}$ and set $T(0,0, \ldots, 0) \leftarrow 0$.

For increasing $i \leq n$, compute the table for each $\left(d_{1}, \ldots, d_{\delta}\right) \in\{0, \ldots, n\}^{\delta}$ as follows. If $A[i]=\sum_{j=1}^{\delta} d_{j} \cdot j$ and $A[i]>A[i-1]$, then set

$$
T\left(i, d_{1}, \ldots, d_{\delta}\right) \leftarrow \min _{d_{1}^{\prime} \leq d_{1}, \ldots, d_{\delta}^{\prime} \leq d_{\delta}}\left(T\left(i-1, d_{1}^{\prime}, \ldots, d_{\delta}^{\prime}\right)+\sum_{j=1}^{\delta} d_{j}-d_{j}^{\prime}\right)
$$

If $A[i]=\sum_{j=1}^{\delta} d_{j} \cdot j$ and $A[i]<A[i-1]$, then set

$$
T\left(i, d_{1}, \ldots, d_{\delta}\right) \leftarrow \min _{d_{1}^{\prime} \geq d_{1}, \ldots, d_{\delta}^{\prime} \geq d_{\delta}} T\left(i-1, d_{1}^{\prime}, \ldots, d_{\delta}^{\prime}\right) .
$$

Otherwise, set

$$
T\left(i, d_{1}, \ldots, d_{\delta}\right) \leftarrow \infty
$$

The correctness of the initialization follows directly from the table definition. For the remaining computation we can thus assume that there is some $i$ such that all entries $T\left(i^{\prime}, d_{1}, \ldots, d_{\delta}\right)$ with $\left(d_{1}, \ldots, d_{\delta}\right) \in\{0, \ldots, n\}^{\delta}$ and $i^{\prime}<i$ were computed correctly.

As discussed above, we interpret an explanation of $A[1, \ldots, i]$ as extension of an explanation for $A[1, \ldots, i-1]$. There are exactly two groups of segments covering position $i$ : those also covering position $i-1$ and those starting at position $i$. Let the set of segments covering position $i$ be described by $\left(d_{1}, \ldots, d_{\delta}\right)$ such that $A[i]=\sum_{j=1}^{\delta} d_{j} \cdot j$ and $A[i]>A[i-1]$. Due to Lemma 1 , no segment ends at position $i$, but since $A[i]>A[i-1]$ at least one new segment has to start at position $i$. By setting $\left(d_{1}^{\prime}, \ldots, d_{\delta}^{\prime}\right)$ such that $d_{j}^{\prime} \leq d_{j}, 1 \leq j \leq \delta$, one considers all possible extensions for explanations of $A[i-1]$ such that no segment ends at position $i$. Clearly, $\sum_{j=1}^{\delta} d_{j}-d_{j}^{\prime}$ further segments have to start at position $i$ to explain $A[i]$. Hence, assignment (4) is correct.

Now, let the set of segments covering position $i$ be described by $\left(d_{1}, \ldots, d_{\delta}\right)$ such that $A[i]=\sum_{j=1}^{\delta} d_{j} \cdot j$ and $A[i]<A[i-1]$. By Lemma 1 no new segment starts at position $i$. The algorithm considers all possible explanations where some 
segments end at position $i$ and the other segments survive to explain $A[i]$. Thus, assignment (5) is correct.

For a given $\left(d_{1}, \ldots, d_{\delta}\right) \in\{0, \ldots, \delta\}$, to find an explanation for $A[1, \ldots, i]$ such that $A[i] \neq \sum_{j=1}^{\delta} d_{j} \cdot j$ is impossible because such explanation does not explain position $i$. Thus the assignment (6) is correct.

The table size is upper-bounded by $n^{\delta+1}$. The trivial upper bound of $O\left(n^{\delta}\right)$ for computing each table entry already leads to a running time of $O\left(n^{2 \delta+1}\right)$. However, the number of entries that have to be considered is smaller. For assignment (4), one only has to consider those entries of Table $T$ that do not have value $\infty$. Hence, $\sum_{j=1}^{\delta}\left|d_{j}-d_{j}^{\prime}\right| \leq|A[i]-A[i-1]| \leq \delta$. This implies that for each table entry the number of previous entries that have to be considered in the minimization is upper-bounded by the number of different multisets that sum up to $\delta$ and thus by $O\left(e^{\pi \sqrt{\frac{2}{3} \delta}}\right)$ [2]. A similar argument applies for assignment (5). The overall running time follows.

\section{A.3 Proof of Theorem 4}

Theorem $4 \mathrm{VE}^{+}$and $\mathrm{VE}$ can be solved in $O(k ! \cdot k+n)$ time. Any instance of $\mathrm{VE}^{+}$or $\mathrm{VE}$ can be reduced in $O(n)$ time to an equivalent one with at most $(2 k-1)$ entries.

Proof. We start with the algorithm for $\mathrm{VE}^{+}$which works as follows. After exhaustive application of Rule 1 branch over all possible segments covering the last entry. Due to Theorem 1(6), it suffices to search for exactly one segment starting at one of the upticks and ending at the last entry. For each branch assign the value $A[n]$ of the last entry as weight to the segment and solve the instance consisting of the remaining entries recursively. To this end, decrease each of the entries covered by the segment by $A[n]$ each, and decrease $k$ by one. Whenever an entry gets negative discard the branch.

Clearly, the search tree produced by the algorithm has depth at most $k$. In the $i$-th level of the search tree, one branches over at most $k+1-i$ upticks (Theorem 1(6)). After exhaustively applying Rule 1 once in $O(n)$ time, the steps performed in each search tree node take $O(k)$ time. The overall running time thus is $O(k ! \cdot k+n)$.

For VE we first apply Theorem 1(4) to transform our instance into a singlepeaked instance (this is necessary to use Theorem 1(6)). The rest works analogously to $\mathrm{VE}^{+}$.

\section{A.4 Proof of Theorem 5}

Towards proving Theorem 5 we briefly discuss some combinatorial properties of clean and messy positions as defined in Definition 4.

Lemma 2. Let $(A, k)$ be an instance of $\mathrm{VE}^{+}$with explaining segment set $\mathcal{I}$. Further, let $I=[i, j] \in \mathcal{I}$ be a clean segment. If there is a clean position $\ell$ such that $i \leq \ell \leq j$ and both $\ell$ as well as $j$ have the same downtick size, then there is 
an explanation with a segment set $\mathcal{I}^{\prime}$ such that $\left|\mathcal{I}^{\prime}\right|=|\mathcal{I}|$ and $\mathcal{I}^{\prime}$ contains a clean segment $I^{\prime}=[i, \ell]$ whose weight equals the weight of $I$.

Proof. The lemma trivially holds for $\ell=j$, so assume $\ell<j$ in the following. Since $\ell$ is clean, there is a clean segment $H=[h, \ell]$ whose weight equals the downtick size of $\ell$ and hence the weight of $I$. Consider a segment set $\mathcal{I}^{\prime}$ obtained from $\mathcal{I}$ by replacing $I$ with $I^{\prime}=[i, \ell]$ and $H$ with $H^{\prime}=[h, j]$. The weights remain unchanged. In particular, the weights of $I$ and $H$ are the same as the weights of $I^{\prime}$ and $H^{\prime}$. In order to show that this is indeed an explanation, we consider the following two cases.

Case 1: $h<i$. We show that in the new explanation for each position, the sum of the weights of the segments covering this position are exactly the same as in the old position. Clearly, we only need to consider positions that are covered by either $I$ or $H$ or by both segments. For position $p \in\{h, \ldots, i-1\}$, if $p$ is only covered by segment set $S_{p} \cup\{H\}$ in the old explanation, then $p$ is also only covered by segment set $S_{p} \cup\left\{H^{\prime}\right\}$ in the new explanation. Hence, the sum of weights of $S_{p} \cup\left\{H^{\prime}\right\}$ equals $A[p]$. Similarly, for position $p \in\{i, \ldots, l-1\}$, if $p$ is only covered by segment set $S_{p} \cup\{H, I\}$ in the old explanation then $p$ is also only covered by segment set $S_{p} \cup\left\{H^{\prime}, I^{\prime}\right\}$ in the new explanation. Finally, for position $p \in\{l, \ldots, j-1\}$, if $p$ is only covered by segment set $S_{p} \cup\{I\}$ in the old explanation then $p$ is also only covered by segment set $S_{p} \cup\left\{H^{\prime}\right\}$ in the new explanation. The correctness follows, because $I, I^{\prime}, H$, and $H^{\prime}$ have the same weight.

Case 2: $h>i$. The proof is similar to that of Case 1: A position $p$ that was only covered by $S_{p} \cup\{I\}$ with $H \notin S_{p}$ is now either only covered by $S_{p} \cup\left\{I^{\prime}\right\}$ or only covered by $S_{p} \cup\left\{H^{\prime}\right\}$; a position $p$ that was previously covered by $S_{p} \cup\{I\}$ and $S_{p} \cup\{H\}$ is now covered by $S_{p} \cup\left\{I^{\prime}\right\}$ and $S_{p} \cup\left\{H^{\prime}\right\}$.

Lemma 3. Let $(A, k)$ be a yes-instance of $\mathrm{VE}^{+}$that is reduced with respect to Rule 1. Then, every explanation of $(A, k)$ has at most $2 k^{\prime}$ messy segments and at most $3 k^{\prime}$ messy positions.

Proof. Let $x$ denote the number of messy segments in some arbitrary explanation for $(A, k)$. Since $(A, k)$ is reduced with respect to Rule 1 , every position of $A$ is the starting point or endpoint of some segment. In particular, every messy segment shares at least one endpoint with another messy segment. Hence, there are at most $1.5 x$ messy positions in the explanation. Furthermore, there are at most $2(k-x)$ clean positions. Thus, $n \leq 2(k-x)+1.5 x$ which implies $x \leq 2 k^{\prime}$, and the number of messy positions is at most $1.5 x \leq 3 k^{\prime}$.

Lemma 4. Let $(A, k)$ be a single-peaked instance of $\mathrm{VE}^{+}$. If vector $A$ has an uptick $i$ and a downtick $j$ of the same size, then there is solution containing the segment $[i, j]$ with weight equal to the size of uptick $i$.

Proof. Since $A$ is single-peaked, $(A, k)$ is an equivalent VE instance, and therefore by Theorem 1(2), it is enough to prove the lemma for the case $j=i+1$. Let $s$ be the size of uptick $i$. Consider an explanation $(\mathcal{I}, w)$ with at most $k$ segments 
as provided by Theorem 1(1). Let $\mathcal{I}_{1}=\{[i, b] \mid b>i+1,[i, b] \in \mathcal{I}\}$ and $\mathcal{I}_{2}=\{[a, i+1] \mid a<i,[a, i+1] \in \mathcal{I}\}$. Note that $s=w\left(\mathcal{I}_{2}\right)+w([i, i+1])=$ $w\left(\mathcal{I}_{1}\right)+w([i, i+1])$. If $[i, i+1] \in \mathcal{I}$, then increase its weight to the size of uptick $i$ and shorten the segments in $\mathcal{I}_{2}$ by making them start only at position $i+1$. We claim that this modified set of segments still explains $A$. It is only necessary to check this for $A[i]$. But, as the weight of $[i, i+1]$ was increased by exactly $w\left(\mathcal{I}_{2}\right)$, while segments of total weight $w\left(\mathcal{I}_{2}\right)$ were shortened not to span over $i$, this is clear. In this case we have the same number of segments.

If $[i, i+1] \notin \mathcal{I}$, then we introduce it with weight $s$ and again shorten the segments in $\mathcal{I}_{2}$ to start in $i+1$. By the above argument, this modified set of segments explains $A$. But it has one more segment than $\mathcal{I}$. We want to show that the vector formed by the sum of segments in $\mathcal{I}_{1}$ and the modified segments from $\mathcal{I}_{2}$ can be explained using $\left|\mathcal{I}_{1}\right|+\left|\mathcal{I}_{2}\right|-1$ segments. To be more precise, let $A^{\prime}$ denote the vector formed by setting $A^{\prime}[j]=\sum_{[a, i+1] \in \mathcal{I}_{1} ; a \leq j} w([a, i+1])$ for $j<i$, $A^{\prime}[j]=\sum_{[i, b] \in \mathcal{I}_{2} ; b>j} w([i, b])$ for $j>i$ and $A^{\prime}[i]=\sum_{[a, i+1] \in \mathcal{I}_{1}} w([a, i+1])=$ $\sum_{[i, b] \in \mathcal{I}_{2}} w([i, b])$. We claim that $\left(A^{\prime},\left|\mathcal{I}_{1}\right|+\left|\mathcal{I}_{2}\right|-1\right)$ is a yes-instance.

Observe that there are at most $\left|\mathcal{I}_{1}\right|$ upticks and at most $\left|\mathcal{I}_{2}\right|$ downticks in $A^{\prime}$. Hence, Rule 1 would reduce $A^{\prime}$ to a vector of length at most $\left|\mathcal{I}_{1}\right|+\left|\mathcal{I}_{2}\right|-1$. Such a vector can be trivially explained by $\left|\mathcal{I}_{1}\right|+\left|\mathcal{I}_{2}\right|-1$ segments.

Theorem 5(1) $\mathrm{VE}^{+}$can be solved in $O\left((2 k)^{3 k^{\prime}} \cdot\left(k^{2}+\left(2 k^{\prime}\right) ! \cdot 2 k^{\prime}\right)+n\right)$ time.

Proof. The algorithm works as follows. First, exhaustively apply Rule 1 to reduce the number of positions to at most $2 k$. Then, try all possibilities for choosing messy positions. Now, consider the remaining positions (which are assumed to be clean). Pick an arbitrary remaining uptick $i$ and let $x$ denote the size of the uptick $i$. If the guess is correct, then there should be at least one clean downtick with size $x$. Now, let $j>i$ be the first remaining downtick $j$ whose downtick size also equals $x$. By Lemma 2, if there is a solution in which $i$ and $j$ are clean, then there is also a solution which contains the clean segment $[i, j]$. Accordingly, one can obtain an equivalent instance by setting $A[\ell] \leftarrow A[\ell]-x$ for each $\ell \in[i, j-1]$ and $k \leftarrow k-1$. Since $x$ is the size of uptick $i$ as well as the size of downtick $j$, $A[i-1]=A[i]$ after this reduction. Hence, we can apply Rule 1 in order to "remove" the position $i$ from $A$. A similar argument applies for position $j$. Hence, after this reduction either all positions are messy, or some are clean and there is currently no segment assigned to these positions. Repeat the above procedure until all remaining positions are messy. There are at most $3 k^{\prime}$ such positions. The remaining instance is solved by the algorithm from Theorem 4 .

The running time of this algorithm can be bounded as follows. First, by Lemma 3, there are at most $3 k^{\prime}$ messy positions in a yes-instance that is reduced with respect to Rule 1. Therefore, there are $O\left((2 k)^{3 k^{\prime}}\right)$ possibilities to select these positions. For each such possibility, there are $O(n)$ applications of the reduction based on Lemma 2 and Rule 1; each time these can be performed in $O(n)$ time. Finally, by Theorem 4 the remaining instance can be solved in $O\left(\left(2 k^{\prime}\right) ! \cdot 2 k^{\prime}+3 k^{\prime}\right)$ time. 
Theorem 5(2) $\mathrm{VE}^{+}$is $\mathrm{W}[1]$-hard with respect to $k^{\prime}$.

Proof. We present a parameterized reduction from the SubSET Sum problem.

SubSet Sum

Input: A multiset $X:=\left\{x_{1}, \ldots x_{\ell}\right\}$ of positive integers and two positive integers $y$ and $k$.

Question: Is there a size- $k$ subset $X^{\prime}$ of $X$ such that $\sum_{x_{i} \in X^{\prime}} x_{i}=y$ ?

Subset Sum is W[1]-hard with respect to the solution size $k$ [11]. In the following, we use $t:=\sum_{1 \leq i \leq \ell} x_{i}$ to denote the total sum of the integers in $X$. Note that by modifying the $x_{i}$ 's we can assume that for every size- $(k-1)$ subset $X^{\prime}$ the sum $\sum_{x_{i} \in X^{\prime}} x_{i}$ is less than $y$ : adding $t$ to each input integer, and $k \cdot t$ to $y$ results in an instance for which this holds. Next, we describe the parameterized reduction.

The input vector $A$ has length $2 \ell+1$. For $i \leq \ell$, we set $A[i]:=\sum_{j=1}^{i} x_{j}$. Let $A[\ell+1]=t-y$. For $i \geq \ell+1$, we set $A[i]=A[2 \ell+2-i]$. The number of allowed segments is set to $\ell+k$. Consequently, $k^{\prime}=2(\ell+k)-(2 \ell+1)=2 k-1$.

We complete the proof by showing that

$(X, y, k)$ is a yes-instance of Subset $\operatorname{Sum} \Leftrightarrow(A, \ell+k)$ is a yes-instance of $\mathrm{VE}^{+}$.

" $\Rightarrow$ ": Let $X^{\prime}$ be a size- $k$ subset of $X$ whose values sum up to $y$. Then, consider the following set $\mathcal{I}$ of segments.

For each $x_{i} \notin X^{\prime}$, add the segment $J_{i}=[i, 2 \ell+3-i]$. There are $\ell-k$ such segments. For each $x_{i} \in X^{\prime}$, add two segments $I_{i}=[i, \ell+1]$ and $I_{i}^{\prime}=[\ell+2,2 \ell+$ $3-i]$. Each of these two types of segments is of size $k$. Hence, $|\mathcal{I}|=\ell+k$. For each $1 \leq i \leq \ell$ set the weights of the segments $J_{i}, I_{i}$ and $I_{i}^{\prime}$ to $x_{i}$. Now, $\mathcal{I}$ explains $A$ : First, for each $i \leq \ell, A[i]=\sum_{j<i} x_{j}$ is explained by $\left\{J_{j} \mid j \leq i \wedge x_{j} \notin X^{\prime}\right\} \cup\left\{I_{j} \mid\right.$ $\left.j \leq i \wedge x_{j} \in X^{\prime}\right\}$. Second, $A[\ell+1]=t-y$ is explained by exactly the segments $J_{j}$ with $x_{j} \notin X^{\prime}$. Finally, for $i>\ell+1, A[i]=A[2 \ell+2-i]=\sum_{j \leq 2 \ell+2-i} x_{j}$ is explained by $\left\{J_{j} \mid j \leq 2 \ell+2-i \wedge x_{j} \notin X^{\prime}\right\} \cup\left\{I_{j}^{\prime} \mid j \leq 2 \ell+2-i \wedge x_{j} \in X^{\prime}\right\}$.

" $\Leftarrow$ ": Let $\mathcal{I}$ be a set of $\ell+k$ segments that explain $A$. By Lemma 1 we can assume that every segment of $\mathcal{I}$ starts at an uptick and ends at a downtick. First, note that for each position $i \leq \ell$, there is at least one segment that starts at $i$. Also, each of these segments has a weight of at most $x_{j}$ for some $x_{j} \in X$. Since $\sum_{x_{i} \in X^{\prime}} x_{i}<y$ for $\left|X^{\prime}\right|<k$ and the size of downtick $\ell+1$ is $y$, at least $k$ segments end at $\ell+1$. Similarly, for each $i \geq \ell+3$ there is at least one segment that ends at position $i$. Each of these segments has a weight of at most $x_{j}$ for some $x_{j} \in X$. Further, since the size of uptick $\ell+2$ is $y$, at least $k$ segments begin at $\ell+2$. This implies that there are exactly $\ell$ segments starting in the first $\ell$ positions and exactly $k$ segments ending at position $\ell+1$. Therefore, for each $i \leq \ell$ there is exactly one segment starting at $i$ which has weight $x_{i}$. Since $k$ of these segments end at position $\ell+1$, they correspond to a size- $k$ set $X^{\prime} \subseteq X$. Finally, the sum $\sum_{x_{i} \in X^{\prime}} x_{i}$ of the integers in this set is exactly $y$, since $A[\ell]=t$ and $A[\ell+1]=t-y$. 
Theorem 5(3) Any single-peaked instance of $\mathrm{VE}^{+}$and any instance of $\mathrm{VE}$ can be reduced in $O\left(k^{2}+n\right)$ time to an equivalent one with most $3 k^{\prime}$ entries. Both problems are solvable in $O\left(\left(2 k^{\prime}\right) ! \cdot 2 k^{\prime}+3 k^{\prime}+k^{2}+n\right)$ time.

Proof. Due to Theorem 1(4), we can assume that the given instance of VE is single-peaked. Also, because of Theorem 1(3), we only need to investigate whether the given single-peaked instance is a yes-instance for $\mathrm{VE}^{+}$. Now, we first apply Rule 1 exhaustively. After that, if there is an uptick and a downtick of the same size, then by Lemma 4 there is an optimal solution containing a segment starting at the uptick and ending at the downtick of weight equal to the size of the uptick. Hence one can subtract such a segment from the vector, decrease $k$ by one, and again reduce the vector using Rule 1 . Note that the length of the vector is reduced by two, while $k$ is reduced by one, so $k^{\prime}$ stays the same. Repeat this procedure until each uptick has size different from each downtick. Now, obviously all positions are messy and by Lemma 3 there are at most $3 k^{\prime}$ messy positions and $2 k^{\prime}$ messy segments explaining them. Thus, one ends up with a problem kernel having at most $3 k^{\prime}$ positions.

The initial applications of Rule 1 take $O(n)$ time. Clearly, at most $k$ upticks and at most $k$ downticks remain. Given an uptick, to find a downtick of the same size and, if successful, subtracting a segment of the corresponding size and applying Rule 1 twice takes $O(k)$ time. Hence, computing the kernel takes $O\left(k^{2}+n\right)$ time. By Theorem 4, the remaining problem kernel can be solved in $O\left(\left(2 k^{\prime}\right) ! \cdot 2 k^{\prime}+3 k^{\prime}\right)$ time.

\section{A.5 Proof of Theorem 6}

Theorem 6(1) $\mathrm{VE}^{+}$and $\mathrm{VE}$ are NP-hard even if $k=n-1$ and every yes-instance has an explanation of at most $k$ segments where each position is covered by at most two segments and each segment has length at most three.

Proof. We reduce from the NP-hard PARTition problem [15].

\section{PARTITION}

Input: A multiset of positive integers $S=\left\{a_{1}, \ldots, a_{n}\right\}$.

Question: Is there a subset $S^{\prime} \subseteq S$ such that $\sum_{a_{i} \in S^{\prime}} a_{i}=\sum_{a_{i} \in S \backslash S^{\prime}} a_{i}$ ?

Given an instance $S=\left\{a_{1}, \ldots, a_{n}\right\}$ of PARTition, we create an instance $(A, k)$ of $\mathrm{VE}$, where $A$ is a vector of length $n^{\prime}=3 n+1$ and $k=3 n$. Namely, $A^{T}$ 
is the vector

$$
\left(\begin{array}{c}
1 \\
2 \\
2+(n+1) a_{1} \\
3+(n+1) a_{1} \\
4+(n+1) a_{1} \\
4+(n+1)\left(a_{1}+a_{2}\right) \\
\vdots \\
2 j-1+(n+1) \sum_{i=1}^{j-1} a_{i} \\
2 j+(n+1) \sum_{i=1}^{j-1} a_{i} \\
2 j+(n+1) \sum_{i=1}^{j} a_{i} \\
\vdots \\
2 n-1+(n+1) \sum_{i=1}^{n-1} a_{i} \\
2 n+(n+1) \sum_{i=1}^{n-1} a_{i} \\
2 n+(n+1) \sum_{i=1}^{n} a_{i} \\
n+(n+1) / 2 \cdot \sum_{i=1}^{n} a_{i}
\end{array}\right)
$$

Obviously, the reduction runs in polynomial time. It remains to show that $S=\left\{s_{1}, \ldots, s_{n}\right\}$ is a yes-instance of Partition $\Leftrightarrow(A, k=3 n)$ is a yes-instance of $\mathrm{VE}^{+}$and $\mathrm{VE}$.

" $\Rightarrow$ ": Let $S^{\prime} \subseteq S$ be a solution for the PARTition instance with $\sum_{a_{i} \in S^{\prime}} a_{i}=$ $\sum_{a_{i} \in S \backslash S^{\prime}} a_{i}$. Further, let $S_{j}^{\prime}:=S^{\prime} \cap\left\{a_{1}, \ldots, a_{j}\right\}, \bar{S}_{j}:=\left\{a_{1}, \ldots, a_{j}\right\} \backslash S^{\prime}$ and $S_{-1}^{\prime}=S_{0}^{\prime}=\bar{S}_{-1}=\bar{S}_{0}:=\emptyset$. We construct the set of segments $\mathcal{I}$ consisting of six subsets and their weights as follows:

$$
\begin{aligned}
& \mathcal{I}_{1}=\left\{[3 j-2,3 j+1] \mid a_{j} \notin S^{\prime}\right\}, \quad \text { with } \quad w([3 j-2,3 j+1])=j+(n+1) \cdot \sum_{a_{i} \in S_{j-1}^{\prime}} a_{i}, \\
& \mathcal{I}_{2}=\left\{[3 j-1,3 j] \mid a_{j} \notin S^{\prime}\right\}, \quad \text { with } \quad w([3 j-1,3 j])=j+(n+1) \cdot \sum_{a_{i} \in \bar{S}_{j-1}} a_{i}, \\
& \mathcal{I}_{3}=\left\{[3 j, 3 j+2] \mid a_{j} \notin S^{\prime}\right\}, \quad \text { with } \quad w([3 j, 3 j+2])=j+(n+1) \cdot \sum_{a_{i} \in \bar{S}_{j}} a_{i}, \\
& \mathcal{I}_{4}=\left\{[3 j-1,3 j+2] \mid a_{j} \in S^{\prime}\right\}, \quad \text { with } \quad w([3 j-1,3 j+2])=j+(n+1) \cdot \sum_{a_{i} \in \bar{S}_{j-1}} a_{i}, \\
& \mathcal{I}_{5}=\left\{[3 j-2,3 j] \mid a_{j} \in S^{\prime}\right\}, \quad \text { with } \quad w([3 j-2,3 j])=j+(n+1) \cdot \sum_{a_{i} \in S_{j-1}^{\prime}} a_{i}, \\
& \mathcal{I}_{6}=\left\{[3 j, 3 j+1] \mid a_{j} \in S^{\prime}\right\}, \\
& \text { with } \quad w([3 j, 3 j+1])=j+(n+1) \cdot \sum_{a_{i} \in S_{j}^{\prime}} a_{i} .
\end{aligned}
$$

As there are exactly three segments for each $a_{j}$, there are $3 n$ segments in total. 
Note that if $a_{j} \notin S^{\prime}$, then $S_{j-1}^{\prime}=S_{j}^{\prime}$. Otherwise $a_{j} \notin \overline{S^{\prime}}$ and $\bar{S}_{j-1}=\bar{S}_{j}$.

Now, we show that $\mathcal{I}$ with weight function $w$ explains the vector $A$. Let $j \in$ $\{1, \ldots, n\}$. At position $3 j-2=3(j-1)+1$ we have $A[3 j-2]=2 j-1+(n+$ 1) $\sum_{i=1}^{j-1} a_{i}$. If $a_{j} \notin S^{\prime}$, then segment $[3 j-2,3 j+1]$ from $\mathcal{I}_{1}$ covers $3 j-2$ and if $a_{j} \in S^{\prime}$, then segment $[3 j-2,3 j]$ from $\mathcal{I}_{5}$ covers $3 j-2$. Both segments have weight $\left(j+(n+1) \sum_{a_{i} \in S_{j-1}^{\prime}} a_{i}\right)$. Additionally, if $a_{j-1} \notin S^{\prime}$, then segment [3(j1), $3(j-1)+2]$ from $\mathcal{I}_{3}$ also covers $3 j-2$ and if $a_{j-1} \in S^{\prime}$, then segment $[3(j-$ 1) $-1,3(j-1)+2]$ from $\mathcal{I}_{4}$ also covers $3 j-2$. In both cases the weight of the segment is $\left((j-1)+(n+1) \sum_{a_{i} \in \bar{S}_{j-1}} a_{i}\right)$. In the former case this holds by definition. In the latter case, since $a_{j-1} \in S^{\prime}$, it holds that $a_{j-1} \notin \overline{S^{\prime}}$ and, thus, ${\overline{S^{\prime}}}_{j-2}={\overline{S^{\prime}}}_{j-1}$. Summarizing, in each case the weights of the two segments covering position $3 j-2$ sum up to

$$
\left(j+(n+1) \sum_{a_{i} \in S_{j-1}^{\prime}} a_{i}\right)+\left((j-1)+(n+1) \sum_{a_{i} \in \bar{S}_{j-1}} a_{i}\right)=2 j-1+(n+1) \sum_{i=1}^{j-1} a_{i}=A[3 j-2] .
$$

In the same way, at position $3 j-1$, we have $A[3 j-2]=2 j+(n+1) \sum_{i=1}^{j-1} a_{i}$. If $a_{j} \notin S^{\prime}$, then only segments $[3 j-2,3 j+1]$ from $\mathcal{I}_{1}$ and $[3 j-1,3 j]$ from $\mathcal{I}_{2}$ cover and explain this position, since

$$
\left(j+(n+1) \sum_{a_{i} \in S_{j-1}^{\prime}} a_{i}\right)+\left(j+(n+1) \sum_{a_{i} \in \bar{S}_{j-1}} a_{i}\right)=2 j+(n+1) \sum_{i=1}^{j-1} a_{i}=A[3 j-1] .
$$

Otherwise, only segments $[3 j-1,3 j+2]$ from $\mathcal{I}_{4}$ and $[3 j-2,3 j]$ from $\mathcal{I}_{5}$ cover and explain this position, since

$$
\left(j+(n+1) \sum_{a_{i} \in \bar{S}_{j-1}} a_{i}\right)+\left(j+(n+1) \sum_{a_{i} \in S_{j-1}^{\prime}} a_{i}\right)=2 j+(n+1) \sum_{i=1}^{j-1} a_{i}=A[3 j-1] .
$$

Also, at position $3 j$, we have $A[3 j]=2 j+\sum_{i=1}^{j} a_{i}$. If $a_{j} \notin S^{\prime}$, then only segments $[3 j-2,3 j+1]$ from $\mathcal{I}_{1}$ and $[3 j, 3 j+2]$ from $I_{3}$ cover and explain this position since the sum of their weights equals

$$
\left(j+(n+1) \sum_{a_{i} \in S_{j}^{\prime}} a_{i}\right)+\left(j+(n+1) \sum_{a_{i} \in \bar{S}_{j}} a_{i}\right)=2 j+(n+1) \sum_{i=1}^{j} a_{i}=A[3 j] .
$$

This also holds for the case that $a_{j} \in S^{\prime}$. Finally, we have only one segment covering the position $3 n+1$ with weight

$$
n+(n+1) \sum_{a_{i} \in \bar{S}_{n}} a_{i}=n+(n+1) \sum_{a_{i} \in S \backslash S^{\prime}} a_{i}=n+(n+1) \frac{1}{2} \sum_{i=1}^{n} a_{i}=A[3 n+1] .
$$


" $\Leftarrow$ ": Let $\mathcal{I}$ with (possibly negative) weights $w$ be an explanation for vector $A$ with at most $k$ segments. Due to Theorem 1(1), we can assume that each negative segment starts at a downtick and ends at an uptick and each positive segment starts at an uptick and ends at a downtick. Since $A$ has only upticks except for position $3 n+1$ and $3 n+2$, a negative segment would have to start at position $3 n+1$. However, in this case there is no uptick for it to end at. Hence, $\mathcal{I}$ has no negative segment and at each uptick, a positive segment must start. As there are exactly $k=3 n$ upticks, exactly one positive segment starts at every uptick and ends either at position $3 n+1$ or $3 n+2$.

We denote the segment of $\mathcal{I}$ starting at position $3 i$ by $I_{i}$. Obviously, $w\left(I_{i}\right)=$ $(n+1) a_{i}$. Furthermore, there are $2 n$ segments of weight 1 . Now let $S^{\prime}:=\left\{a_{i} \mid\right.$ $I_{i}$ ends at position $\left.3 n+2\right\}$. We show that $S^{\prime}$ is a solution of the PARTition instance $S$ : Let $x \in\{0, \ldots, 2 n\}$ be the number of segments of weight 1 that cover position $3 n+1$. We have $x+(n+1) \sum_{a_{i} \in S^{\prime}} a_{i}=A[3 n+1]=n+(n+1) \frac{1}{2} \sum_{i=1}^{n} a_{i}$. As $|n-x| \leq n$, we have $\sum_{a_{i} \in S^{\prime}} a_{i}=\frac{1}{2} \sum_{i=1}^{n} a_{i}$. Hence, $S^{\prime}$ is a solution for the PARTition instance $S$.

As we can see from the reduction, every yes-instance of PARTITION is reduced to a yes-instance that can be explained by segments with $l=3$ and $o=2$ and every no-instance is reduced to an instance that cannot be explained by segments of any size. The statement of Theorem 6(1) follows.

Theorem 6 (2) Both $\mathrm{VE}^{+}$and $\mathrm{VE}$ can be solved in $O\left(n^{2}\right)$ time for maximum segment length $l=2$.

Proof. We show a dynamic programming algorithm for $\mathrm{VE}^{+}$. Afterwards, we show how to extend our algorithm to VE.

Let $(A, k)$ be an input instance, where $A$ is a vector of length $n$. The last position is only covered by either one length-two segment or one length-one segment, but not both, because then we can always transform it into two lengthone segments. Due to this, if we have an optimal solution for a vector of length $x$, then we can find an optimal solution for a vector of length $x+1$ which contains either an additional length-one segment or a length-two segment covering the last position.

Based on this idea, we use dynamic programming with a table $s$ indexed by $1, \ldots, n$ : For each $j \leq n$, we store in $s[j]$ the minimum number of segments needed to explain the subvector $A[1, \ldots, j]$. We let $s[0]=0$ for simplicity. We start with $j=1$. For $j=1$, we set $s[1]=1$ which is obviously correct. Now assume that for an index $j \leq n, s[i]$ was already computed for each $i<j$ and we now compute $s[j]$. We begin with $i:=j$ and $a_{i}^{j}:=A[j]$. We set

$$
a_{i-1}^{j}:=A[i-1]-a_{i}^{j} \text { and } i:=i-1
$$

as long as

$$
a_{i}^{j}>0 \text { and } i>1 .
$$

The idea behind this computation is that if we want to add some length-two segment with weight $a_{i}^{j}$ covering position $i$ and $i-1$, then we should make sure 
that after this the remaining value at position $i-1$ is non-zero, since otherwise it is better to just cover position $i$ separately. If Condition $\left(^{*}\right)$ does not hold, then there are two cases: If $a_{i}^{j}=0$, then let $s[j]:=\min \{s[j-1]+1, s[i-1]+j-i\}$; otherwise let $s[j]:=s[j-1]+1$. Finally, once the table is completed, we answer yes if $s[n] \leq k$, and no otherwise.

As the algorithm obviously works in $O\left(n^{2}\right)$ time, it remains to show that the algorithm fills the table correctly. The proof is by induction on $j$. Obviously $s[1]$ is computed correctly. For $j \leq n$, assume $s[i]$ is optimal for all $i<j$. We show that $s[j]$ is also optimal.

Let us first show that there is an explanation for $A[1, \ldots, j]$ with $s[j]$ segments. We have two cases: If $s[j]=s[j-1]+1$, then we use the explanation for $A[1, \ldots, j-1]$ with $s[j-1]$ segments and add a single length-one segment to explain $A[j]$. Otherwise, there is an $i \in\{1, j-1\}$ such that $s[j]=s[i-1]+j-i$. Let $a_{j}^{j}:=A[j]$, and $a_{x}^{j}:=A[x]-a_{x+1}^{j}, i \leq x \leq j-1$. Note that $a_{i}^{j}=0$ because of Condition $(*)$. Then, we use the explanation for $A[1, \ldots, i-1]$ with $s[i-1]$ segments and add a set $\mathcal{I}$ of $j-i$ segments of length two such that for each $z \in\{i, \ldots j-1\}$, we have a segment $I_{z}=[z, z+2]$ with weight $a_{z+1}^{j}$. Clearly, positions from 1 to $i-1$ are already explained. Since $a_{i}^{j}$ equals zero, we have $A[i]=a_{i+1}^{j}$ which is also the weight of $I_{i}$. Thus, $\mathcal{I}$ explains $A[i]$. For $z \in\{i+1, \ldots, j-1\}$, we have $A[z]=a_{z}^{j}+a_{z+1}^{j}$ and $A[j]=a_{j}^{j}$. Hence, the subvector $A[i+1, \ldots, j]$ is also explained by $\mathcal{I}$.

Next, we show that $s[j]$ is optimal. Assume that there is an explanation $(\mathcal{I}, w)$ of $A[1, \ldots, j]$ with $r$ segments. We will show that $r \geq s[j]$. Without loss of generality, we can assume that every length-one segment exclusively covers a position, since otherwise, we can either merge two length-one segments or split one length-two segment into two length-one segments and merge one of them with the original length-one segment. Let $i$ be the last position such that all segments in $\mathcal{I}$ covering $i$ start at $i$. If $i=j$, then $\mathcal{I} \backslash\{[j, j+1]\}$ is an explanation for $A[1, \ldots, j-1]$, and $r \geq s[j-1]+1 \geq s[j]$ as $s[j-1]$ is optimal. If $i<j$, then $\mathcal{I}$ contains a chain of $j-i$ overlapping length-two segments $I_{i+1}=[i, i+2], \ldots, I_{j}=[j-1, j+1]$ starting at $i$ and ending at $j+1$. Since these are the only segments explaining positions $i, \ldots, j$, their weights are $w\left(I_{j}\right)=A[j]$ and $w\left(I_{z}\right)=A[z]-w\left(I_{z+1}\right), j-1 \geq z \geq i+1$. Position $i$ is only explained by $I_{i+1}$, so we have $A[i]=w\left(I_{i+1}\right)=A[i+1]-w\left(I_{i+2}\right)$. Note that applying the dynamic programming algorithm $a_{z}^{j}=w\left(I_{z}\right), i+1 \leq z \leq j$. This means the algorithm stops at position $i$ with $a_{i}^{j}=w\left(I_{i+1}\right)-a_{i+1}^{j}=0$. Thus, $s[j]=\min \{s[j-1]+1, s[i-1]+j-i\} \leq s[i-1]+j-i$. Furthermore, $\mathcal{I} \backslash\left\{I_{z} \mid z \in\{i+1, \ldots j-1\}\right\}$ is an explanation for $A[1, \ldots, i-1]$. Hence, $r \geq s[i-1]+j-i \geq s[j]$ because $s[i-1]$ is optimal.

To solve VE, it is enough to change Condition $(*)$ in the loop of the above algorithm to "While $a_{i}^{j} \neq 0$ and $i>1 \ldots$ " The rest of the proof remains the same. 